Revista de Antropología Social

ISSN: 1131-558X

https://dx.doi.org/10.5209/raso.65621

\title{
Conflicto y paz: una lectura generizada de la participación política transnacional colombiana
}

Bermúdez, Anastasia. 2016. International Migration, Transnational Politics and Conflict: The Gendered Experiences of Colombian Migrants in Europe. Londres: Palgrave Macmillan

Si bien las migraciones internacionales se han convertido en una de las principales características de nuestras sociedades contemporáneas, sus causas y consecuencias son tanto heterogéneas como variables a lo largo del tiempo. El libro que aquí se reseña es una valiosa contribución que nos permite debatir acerca de uno de los principales $-\mathrm{y}$ recientes- focos de interés que desde las ciencias sociales han surgido en lo referente a los movimientos migratorios: las prácticas políticas transnacionales. Anastasia Bermúdez, investigadora contratada Ramón y Cajal en la Universidad de Sevilla y colaboradora científica en el Center for Ethnic and Migraton Studies (CEDEM) en la Universidad de Liège, Bélgica, publica esta obra fruto de más de veinte años de investigación sobre diferentes aspectos de la migración colombiana a Europa. El interés acerca de esta temática por parte de la autora surge tras varios encuentros informales y formales con migrantes latinoamericanos y colombianos en el Reino Unido durante los 90, que posteriormente también se darían en España y Bélgica y que fueron motivados por "la falta de atención en la academia hacia la agencia de los migrantes colombianos en los procesos de paz" (p. 2). Estos encuentros dieron lugar a la realización de la tesis doctoral de la autora, así como a varias investigaciones postdoctorales sobre la materia.

La investigación presentada y desarrollada en el libro se dividió en varias fases cronológico-espaciales. En 1995 completó su primer proyecto cualitativo en la Queen Mary University of London, en el marco de sus estudios de licenciatura en Geografía y Política, analizando la migración de mujeres colombianas y peruanas a Londres. Este primer acercamiento exploratorio le llevó a detectar el conflicto en Colombia como uno de los factores de expulsión y de configuración de este flujo migratorio. Años después, en el 2002-2003, llevó a cabo otro pequeño proyecto durante su máster en Geografía, también en la Queen Mary University of London, centrado en las experiencias de refugiados colombianos en Londres desde una perspectiva de género, lo que en 2008 se materializaría en una investigación dentro del programa doctoral en Geografía - realizado en la misma universidad - enfocada a las políticas transnacionales generizadas en Reino Unido y España en relación con el conflicto armado y la perspectiva de la consecución de la paz. Todos estos estudios se completarían con otras investigaciones centradas en la participación política local-transnacional de migrantes latinoamericanos en Andalucía, Madrid y Barcelona, en el "voto exterior" de colombianos en Londres y Madrid, y finalmente, en el 2014-2015, culminaría alrededor de 20 años de investigación con un estudio sobre el impacto de la crisis económica europea en las experiencias sobre la integración de migrantes colombianos en Madrid, Londres y Bruselas. En cuanto a la metodología empleada, la autora se sirvió de técnicas mixtas, tanto cuantitativas como cualitativas, dentro 
de las cuales pueden distinguirse los cuestionarios y las entrevistas semiestructuradas -de los cuales realizó 800 y 200, respectivamente-, y también la observación participante y el análisis de documentos y sitios web.

La obra publicada se centra en tres aspectos que, según la autora, han atraído la atención pública, académica y política. El primero es "cómo la migración internacional impacta en la participación política y en la emergencia de campos políticos transnacionales" (p. 1), el segundo es "el impacto que los conflictos armados tienen en los flujos migratorios y cómo se relacionan las diásporas con el conflicto y los esfuerzos por la consecución de la paz en el país de origen" (p. 1) y, el tercero es "el rol que el género juega en la migración, el conflicto y la paz" (p. 1). Estos ejes se articulan a lo largo del libro estructurados en tres partes. La Parte I es un marco conceptual y contextual en el que se ahonda en el bagaje teórico que deriva de la investigación en tres campos interconectados: la migración transnacional, la participación política transnacional de los migrantes y el conflicto armado y la paz en el contexto de la migración (p. 17). En este apartado se exploran las corrientes actuales dentro de la investigación teórica y empírica sobre los estudios migratorios en la Sociología, las Ciencias Políticas, la Geografía y la Antropología Social, además de ofrecer nuevas evidencias y contribuciones conceptuales. Estas aportaciones parten de una revisión y discusión crítica de temas claves, como son la aplicación de la perspectiva transnacional al estudio de la migración internacional y de las diásporas, la discusión en torno a la implicación política de migrantes a nivel diaspórico o transnacional y alrededor de cómo una perspectiva de género y un acercamiento interseccional pueden ser integrados para proveer una perspectiva más holística del fenómeno. Por otro lado, el capítulo que cierra el primer bloque está dedicado a contextualizar la heterogeneidad de la migración colombiana, interna y externa - esta última a Reino Unido, España y Bélgica, los destinos en los que se centra su investigación-, siendo tenidos en cuenta factores tanto socioeconómicos como políticos, además del papel del conflicto armado y los esfuerzos por la consecución de la paz. La Parte II se centra en la variedad de trayectorias migratorias y de conexiones transnacionales de las comunidades migrantes colombianas. Se profundiza en las experiencias específicas de refugiados y solicitantes de asilo colombianos en Europa, debido a su importante papel dentro de las políticas diaspóricas y, finalmente, se centra en lo que los migrantes identifican como los asuntos más relevantes durante el asentamiento y la integración en la sociedad receptora y que dan forma a su participación política posterior.

Finalmente, la Parte III pone el foco en las prácticas políticas transnacionales de los migrantes colombianos, sobre todo en aquellas que se relacionan con el conflicto armado y los esfuerzos por la construcción de la paz en Colombia. Estas prácticas políticas transnacionales se analizan a partir del rol de los "actores desde arriba" (p.143), como es el Estado colombiano, poniendo en evidencia cómo el transnacionalismo está reconfigurando el estado-nación, que en lugar de desaparecer está llevando a nuevas formas de ciudadanía (Levitt y de la Dehesa, 2003; Collyer y King, 2015). Algunas de las iniciativas estatales y de empresas y fundaciones colombianas creadas con el objetivo de estrechar lazos con la diáspora desarrolladas en estos capítulos son Colombia Nos Une (CNU) y Conexión Colombia (CC). También se ejemplifican los "actores desde abajo" (p. 144), analizando la participación de migrantes colombianos en las elecciones y partidos políticos del lugar de origen, sin pasar por alto finalmente el papel de las asociaciones de migrantes, que a menudo implementan una perspectiva local-transnacional y se centran tanto en las "políticas de inmgración" como en las "políticas de emigración" (Ostegaard-Nielsen, 2009). 
Una de las contribuciones de la investigación presentada por Anastasia Bermúdez en esta obra es la amplitud de la definición de "políticas transnacionales" que se maneja, en la que se integran simultáneamente los niveles macro, meso y micro. Además de considerar el rol de los Estados y otros actores con poder en el campo transnacional, se tienen en cuenta también las prácticas transnacionales de individuos, grupos de migrantes y organizaciones e instituciones de la sociedad civil. Como resalta la autora, "estos tres niveles de análisis no están siempre integrados, pero considerarlos a la vez nos permite que diferentes tipos de prácticas políticas sean visibles" (p. 235). Este punto nos remite a las críticas que desde la antropología social se han dirigido a una de las perspectivas hegemónicas en el estudio de la acción colectiva de los migrantes desde la Ciencia Política: la Estructura de Oportunidad Política (POS, por sus siglas en inglés). Si bien la POS pone el foco en los factores institucionales y contextuales que influyen e incluso permiten la participación política de migrantes, su definición de "estructuras" ha sido tachada de rígida, debido a que estas quedan limitadas al aparato institucional de la sociedad de residencia. De hecho, debido a la importancia -en ocasiones sobredimensionada- que se le da al "clima institucional" para explicar la acción colectiva de migrantes, los autores que han aplicado esta perspectiva han sido identificados como "institucionalistas" (Però, 2009). Se ha sugerido, y la obra reseñada es ejemplo de ello, ampliar lo que se entiende por estructura de oportunidad política para incluir ya sea asociaciones de migrantes, como acciones colectivas menos organizadas y no siempre vinculadas con una incidencia política explícita (Landolt y Goldring, 2006). La complejización y amplitud de la POS desde la antropología permite profundizar en la agencia de los migrantes en los procesos y prácticas políticas, además de pensar lo político como algo más allá de lo formal.

Por otro lado, la adopción de una perspectiva interseccional permite a la autora ir más allá del género, la clase y la raza para incluir otras variables específicamente relacionadas con la experiencia migratoria como la edad, el tipo de migración, el estatus legal y socioeconómico, la duración de la residencia o el capital político que los migrantes traen consigo. La interseccionalidad supone un acercamiento a "las múltiples estructuras sociales y procesos que intervienen para producir identidades y posiciones sociales específicas" (Anthias, 2012: 106), que son más que la suma de opresiones y que alumbran la manera en que, en este caso concreto, los colombianos se comportan políticamente, rehuyendo de averiguaciones pre-empíricas que remiten a los hombres a la política formal y a las mujeres a la política informal.

Actualmente, la participación política transnacional de la comunidad migrante está en el punto de mira, ya sea por el impacto económico de las remesas en sus lugares de origen como por su capacidad movilizadora y de transmisión de conocimiento, tecnología y valores, además de su influencia en contextos de conflicto y de construcción de la paz. Investigaciones como la aquí presentada suponen un avance en el conocimiento de los factores complejos que influyen en las experiencias migratorias y la participación política transnacional y en cómo una perspectiva interseccional puede arrojar luz a las situaciones y posiciones específicas que configuran los campos políticos transnacionales.

Cristina Barrial Berbén Universidad Complutense de Madrid cbarrial@ucm.es 


\section{Bibliografía}

Anthias, Floya (2012). "Transnational mobilities, migration research and intersectionality". Nordic Journal of Migration Research, 2(2): 102-110.

Collyer, Michael; King, Russell (2015). "Producing transnational space: International migration and the extra-territorial reach of state power". Progress in Human Geography, 39(2): 185-204.

Landolt, Patricia; Golrdring, Luin (2006). "Activist dialogues and the production of refugee political transnationalism: Chileans, Colombians and non-migrant civil society in Canada”. Paper presentado en II Conferencia de la Red Internacional de Migraciones y Desarrollo, Cocoyoc, México.

Levitt, Peggy; de la Dehesa, Rafael (2003). "Transnational migration and the redefinition of the state: Variations and explanations". Ethnic and Racial Studies, 26(4): 587-611

Østergaard-Nielsen, Eva (2009). "La política a través de las fronteras: reflexiones sobre la dimensión transnacional de la participación política de los migrantes”, en A. Escrivá, A. Bermúdez et al. (eds.), Migración y participación politica. Estados, organizaciones y migrantes latinoamericanos en perspectiva local-transnacional. Madrid: CSIC, 17-41.

Però, Davide (2009). "Las movilizaciones políticas de los latinoamericanos en Londres", en A. Escrivá, A. Bermúdez et al. (eds.), Migración y participación política. Estados, organizaciones y migrantes latinoamericanos en perspectiva local-transnacional. Madrid: CSIC, 155-182. 